\title{
EVALUACIÓN DE LAS PROPIEDADES DINÁMICAS DE UN PÓRTICO PLANO (MDOF) EN UNA MESA VIBRATORIA
}

\section{EVALUATION OF THE DYNAMIC PROPERTIES OF A 2D-FRAME (MDOF) IN A SHAKE TABLE}

\author{
Andrea Tapia Andrade ${ }^{1, *}$ (D), Wilson Torres Berni ${ }^{1}$
}

Recibido: 14-11-2020, Recibido tras revisión: 22-02-2021, Aceptado: 04-03-2021, Publicado: 01-07-2021

\section{Resumen}

Hoy en día, el estudio de la dinámica estructural es fundamentalmente teórico con acceso a ciertas simulaciones vía software, sin embargo, este proyecto intenta que el estudiante pueda entender y observar de manera física las respuestas dinámicas de modelos experimentales. Estos modelos corresponden a pórticos planos de múltiples grados de libertad, que están sometidos a aceleración en la base, la cual es generada por una mesa vibratoria. Los resultados obtenidos se compararán con teóricos. Estos fueron obtenidos basándose en la descomposición modal y en el método de Newmark para el cálculo de la respuesta dinámica, considerando variación lineal en la aceleración de cada piso. La aplicación generada, ATH Dynamic Responses, proporcionó las respuestas teóricas, mediante una interfaz gráfica amigable para el usuario. Los modelos experimentales están constituidos por dos materiales: acero inoxidable (parantes) y láminas de acrílico (pisos), y fueron ensayados sobre una mesa vibratoria. La adquisición de datos se realizó mediante acelerómetros que se colocaron en cada piso y sobre la mesa vibratoria, fueron corregidos, tanto por línea base como con el filtro pasa bajo. Los resultados obtenidos muestran que la instrumentación con una mesa vibratoria y adquisición de datos con acelerómetros proporcionan valores muy similares a los teóricos en cuanto a respuestas dinámicas y propiedades modales.

Palabras clave: descomposición modal, dinámica estructural, modelo experimental, mesa vibratoria, Newmark, filtro

\section{Abstract}

At present, the study of structural dynamics is mainly theoretical with access to certain simulations through software, however, this project attempts that the student may understand and physically observe the dynamic responses of experimental models. These models correspond to $2 \mathrm{D}$ - frames with multiple degrees of freedom that are subjected to acceleration in the base; this acceleration is generated by a Shake Table II, and the results obtained will be compared with theoretical results. These theoretical results were obtained based on modal decomposition and Newmark's method for calculating the dynamic response, considering the linear variation in the acceleration of each floor. The application developed, ATH Dynamic Responses, provided the theoretical responses through a graphical interface friendly for the user. The experimental models are constituted by two materials: stainless steel for frame legs and acrylic sheets for floors; these were tested on a Shake Table II". The data was acquired using accelerometers that were placed in each floor and in the shake table, and they were corrected both by baseline and with the low pass filter. The results obtained show that the instrumentation with the Shake Table II and the data acquisition with accelerometers provide results similar to the theoretical ones regarding dynamic responses and modal properties.

Keywords: modal decomposition, structural dynamics, experimental model, Shake Table, Newmark, filter

\footnotetext{
$\overline{1, *}$ Carrera de Ingeniería Civil, Universidad Politécnica Salesiana, Quito, Ecuador

Autor para correspondencia atapiaa@est.ups.edu.ec.
}

Forma sugerida de citación: Tapia Andrade, A. y Torres Berni, W. (2021). «Evaluación de las propiedades dinámicas de un pórtico plano (MDOF) en una mesa vibratoria». InGENIUs. N. ${ }^{2}$ 26, (julio-diciembre). pp. 49-62. DOI: https: //doi.org/10.17163/ings.n26.2021.05. 


\section{Introducción}

Ecuador tiene una actividad sísmica considerable, el último sismo que afectó en gran intensidad al país ocurrió el 16 de abril de 2016 con una magnitud de momento $\mathrm{M}_{\mathrm{w}} 7.8$, sucedió en las provincias costeras de Manabí y Esmeraldas [1], dejando daños materiales, colapsos en edificaciones y cerca de setecientos muertos.

El terremoto se originó debido a la subducción de la placa oceánica de Nazca por debajo de la placa sudamericana; el rozamiento entre ambas placas produce acumulación de energía elástica, la cual se relaja cuando existe una ruptura súbita y se produce el evento sísmico [2].

Esto ha motivado a los ingenieros a desarrollar una filosofía que se centre en impedir la pérdida de vidas, controlando el colapso de todas las estructuras [3]. Por lo cual toda edificación debe ser diseñada considerando la solicitación sísmica; el análisis de la estructura frente a este tipo de carga es el principal problema que intenta resolver la dinámica estructural.

En la mayoría de los casos, el estudio de la dinámica estructural se lo realiza de manera teórica, sin poder observar físicamente el comportamiento de las estructuras ante un sismo o aceleración en la base. Por lo cual este proyecto se centra en construir modelos experimentales de múltiples grados de libertad con materiales apropiados, eso significa losas muy rígidas comparadas con los pilares a flexión, donde el modelo va a concentrar la deformación de la estructura. Además, tanto los pilares como los pisos se consideran axialmente rígidos, en el modelo analítico, se va a considerar que los pisos concentran la masa, y que los parantes son quienes colaboren con la rigidez, es decir, que se comporte como un edificio a corte [4].

Para obtener respuestas dinámicas y propiedades modales en modelos experimentales y estructuras reales se han implementado técnicas experimentales, como la colocación de acelerómetros triaxiales, o la utilización de equipos de excitación dinámicas que reproducen sismos a escala [5]. En el proyecto se utilizó una mesa vibratoria, conocida como Shake Table II [6]. Este dispositivo es un simulador de terremotos para modelos físicos académicos pequeños (masa máxima $=$ $7,5 \mathrm{~kg}$ ), que permitirá generar aceleraciones de piso en forma de pulsaciones, barridos sinusoidales y registros sísmicos (escalados) [6]. La Shake Table II permite reproducir aceleraciones de piso en dos direcciones (x, y), sin embargo, para el proyecto se trabajó con modelos planos, se utilizó aceleración unidireccional, cada piso solo se moverá en una dirección [7].

En $[8,9]$ y $[10]$ se han determinado propiedades dinámicas de estructuras experimentales con el mismo método, sin embargo, estas se centran solo en desplazamientos máximos y otras solo en la obtención de frecuencias, además, están limitados a dos pisos. En el proyecto se analizará desde la obtención de frecuencias, amortiguamientos y, además, los modelos por su modo de construcción, tienen la posibilidad de apilarse y llegar hasta un modelo de seis pisos, siendo la única limitante, el peso máximo que soporte la mesa vibratoria.

\section{Materiales y métodos}

En el proyecto consta del sustento teórico, donde se da a conocer los conceptos de la dinámica estructural utilizados para el desarrollo del script en MatLab; y la parte experimental, donde se establece el tipo de material a utilizarse, las características geométricas, la aceleración de la base, la aceleración de cada piso, la operación de la mesa vibratoria, la forma de adquirir respuestas y su procesamiento.

\subsection{Sistema de múltiples grados de libertad}

Un sistema de múltiples grados de libertad (MDOF) se puede entender desde el punto de vista de la dinámica estructural como aquel que requiere más de una coordenada para describir su movimiento. Los grados de libertad también pueden determinar las direcciones de la aceleración de las masas concentradas. En el proyecto, la dirección del sismo será uniaxial, por lo tanto, el número de grados de libertad, será uno por cada piso [7].

Los modelos experimentales son modulares y pueden llegar a sistemas desde uno hasta seis grados de libertad, los ensayos fueron realizados con los sistemas de uno, dos y tres grados de libertad. En la Figura 1 se muestra el modelo de tres grados de libertad.

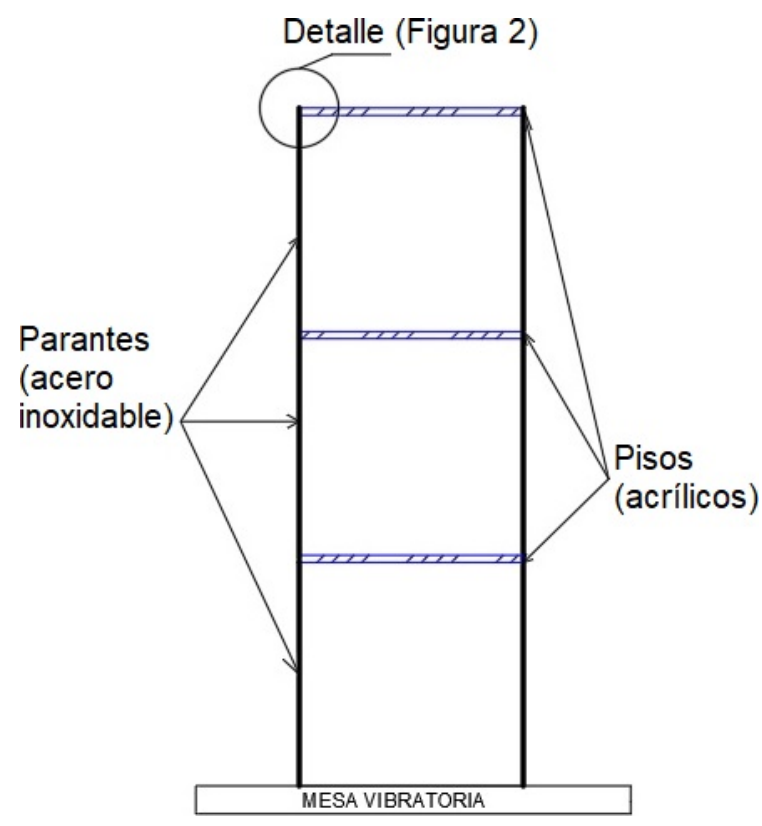

Figura 1. Modelo de tres grados de libertad 


\subsection{Edificio a cortante}

Un edificio a cortante se caracteriza como un arreglo que concentra la masa en cada piso, además, deben actuar como diafragmas infinitamente rígidos a flexión y carga axial. Por lo tanto, en el modelo, solo las columnas deben colaborar con la rigidez [4].

Las consideraciones realizadas anteriormente permiten simplificar la estructura y resolver el problema como un MDOF, donde las losas infinitamente rígidas, permiten asegurar que no existirán rotaciones entre parantes y pisos. En el modelo experimental la rotación se controló en función de la conexión que tiene el parante y el acrílico, ya que la conexión no está fija en un punto, sino en un área como se observa en la Figura 2. En cuanto a la deformación axial, será despreciable, debido a las características físicas de los pisos.

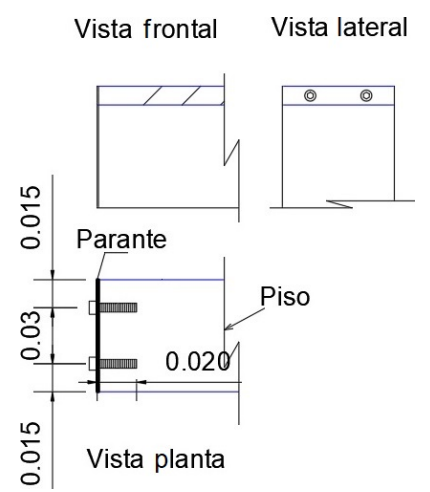

Figura 2. Detalle de conexión

En el modelo experimental, se debe tener en cuenta que las columnas aportan masa, por lo cual se realizó la consideración de que la losa concentra la mitad de la masa de cada columna que esta sobre y bajo de ella, como se muestra en la Figura 3.

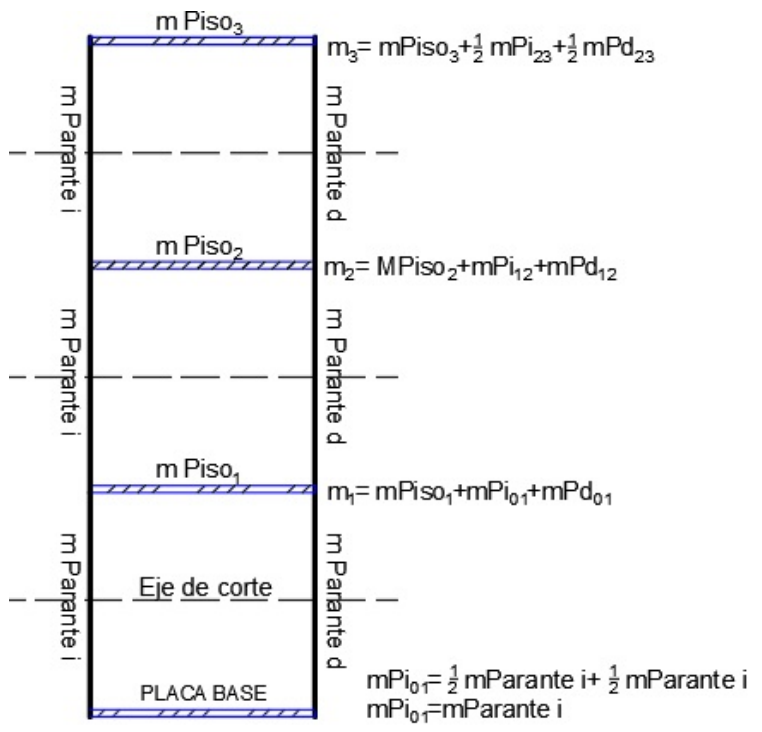

Figura 3. Distribución de masa de la estructura
Donde: $\mathrm{mPi}_{\mathrm{ij}}$ y $\mathrm{mPd}_{\mathrm{ij}}$ es la masa del parante desde el grado de libertad i hasta el grado de libertad j.

Eje de corte: es el que ayuda a cuantificar cómo se concentró la masa de cada piso.

\subsection{Fuerza inercial}

Relaciona las fuerzas externas que actúan sobre la masa de la estructura con las aceleraciones de los grados de libertad dinámicos como se muestra en la Ecuación (1) [11].

$$
\{F i\}=[M] \times\{\ddot{x}\}
$$

Donde $\{F i\}$ es el vector de la fuerza de inercial, $[M]$ es matriz de masa y $\{\ddot{x}\}$ es el vector de aceleración de los grados de libertad.

La matriz de masa (2) es una estimación de la masa de toda la estructura, a esta matriz también se la conoce como «matriz de masa concentrada» [12].

$$
[M]=\left[\begin{array}{cccc}
m_{1} & 0 & 0 & 0 \\
0 & m_{2} & 0 & 0 \\
0 & 0 & m_{3} & 0 \\
0 & 0 & 0 & m_{4}
\end{array}\right]
$$

\subsection{Fuerza elástica}

Relaciona las fuerzas externas sobre la rigidez de la estructura con los desplazamientos de los grados de libertad dinámicos, como se muestra en la Ecuación (3) [11].

$$
\{F s\}=[K] \times\{x\}
$$

Donde $\{F s\}$ es el vector de la fuerza elástica, $[K]$ es matriz de rigidez y $\{x\}$ es el vector de desplazamiento de los grados de libertad.

La matriz de rigidez (4) incluye propiedades de las columnas, como son la longitud, área y módulo de elasticidad [12].

$$
[K]=\left[\begin{array}{cccc}
k_{11} & k_{12} & \cdots & k_{1 N} \\
k_{21} & k_{22} & \cdots & k_{2 N} \\
\vdots & \vdots & \vdots & \vdots \\
k_{N 1} & k_{N 2} & \cdots & k_{N N}
\end{array}\right]
$$

\subsection{Fuerza de amortiguamiento}

Es un mecanismo con el cual se puede disipar energía de la estructura, según esto se relacionan las fuerzas externas que actúan sobre el amortiguamiento con las velocidades de los grados de libertad dinámicos como se muestra en la Ecuación (5) [11].

$$
\{F d\}=[C] \times\{\dot{x}\}
$$

Donde $\{F d\}$ es el vector de la fuerza de amortiguamiento, $[C]$ es matriz de amortiguamiento y $\{\dot{x}\}$ es el vector de velocidad de los grados de libertad. 
Para la obtención de la matriz de amortiguamiento se va a suponer amortiguamiento clásico. La matriz de amortiguamiento clásico se puede utilizar en este tipo de modelos, si los mecanismos de amortiguación son similares en toda la estructura, es decir, una estructura que sea de MDOF y que a su vez esté conformada por un mismo sistema estructural y materiales similares, en toda la altura [11].

Uno de los procedimientos dentro del amortiguamiento clásico es el amortiguamiento modal. El análisis provee el amortiguamiento de un número específico de modos, como se indica en la Ecuación (6) [13] y [14].

$$
\left\{\phi_{n}\right\}^{T} \times[C] \times\left\{\phi_{n}\right\}=2 \times \xi_{n} \times \omega_{n}
$$

Donde $C$ es matriz de amortiguamiento, $\phi_{n}$ es el vector de cada forma modal, $\xi_{n}$ es el factor de amortiguamiento, $\omega_{n}$ frecuencia natural, y $n$ es número de modos.

\subsubsection{Factor de amortiguamiento}

Una característica importante es el amortiguamiento de la estructura, el cual queda definido con base en el factor de amortiguamiento, por esta razón hace falta obtenerlo aplicando el método del ancho de banda descrito a continuación [15].

\section{a) Ancho de banda}

Se trata de un método para la obtención del factor de amortiguamiento en el dominio de la frecuencia; esta técnica es ampliamente utilizada en la práctica profesional, en la cual una estructura debe ser excitada por pulsaciones simultáneas o individuales a diferentes frecuencias [16].

Con el fin de aplicar el método se debe considerar el efecto del movimiento de la base, por lo cual se procede a encontrar el cociente entre las amplitudes de la transformada de Fourier de los registros de aceleración de cada losa con respecto a los registros de la base. A esto se lo conoce como transmisibilidad [11], la cual se indica en la Ecuación (7).

$$
\operatorname{Tr}=\frac{\alpha_{o}(\omega)}{\alpha_{b}(\omega)}
$$

Donde $\operatorname{Tr}$ es transmisibilidad, $\alpha_{o}(\omega)$ amplitud de la aceleración de cada piso en el dominio de la frecuencia y $\alpha_{b}(\omega)$ amplitud de la aceleración de la base en el dominio de la frecuencia.

A partir del cálculo de la transmisibilidad se obtienen gráficas respecto de la frecuencia y el factor de amortiguamiento se determina mediante la diferencia entre dos frecuencias llamadas puntos de media potencia sobre la frecuencia correspondiente de cada modo. Los puntos de media potencia son las frecuencias que se encuentran a $\frac{1}{\sqrt{2}}$ de la amplitud máxima de la transmisibilidad, como se indica en la Figura 4, y esto permitirá aplicar la Ecuación (8) [17].

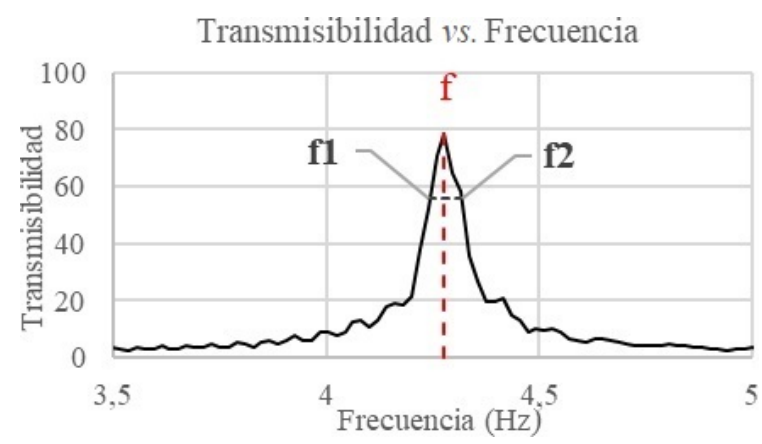

Figura 4. Ancho de banda

$$
\xi=\frac{f_{2}-f_{1}}{2 \times f}
$$

Donde $f$ es la frecuencia con amplitud máxima $(\mathrm{Hz}), f_{1}$ y $f_{2}$ son las frecuencias de media potencia $(\mathrm{Hz})$.

\subsection{Ecuación del movimiento}

Con base en las fuerzas detalladas en las secciones anteriores, se establecerá la ecuación del sistema MDOF, tomándose en cuenta la segunda ley del movimiento de Newton, se obtiene la Ecuación (9).

$$
[M] \times\{\ddot{u}\}+[C] \times\{\dot{u}\}+[K] \times\{u\}=-[M] \times\{\iota\} \times\{\ddot{x} o\}
$$

Donde $\{\iota\}$ es el vector de colocación, $\{\ddot{x} o\}$ la aceleración de piso para cada instante de tiempo y $u$ es la coordenada relativa de cada grado de libertad respecto de la base.

En la Figura 5 se presentan las fuerzas actuantes, sobre el sistema.

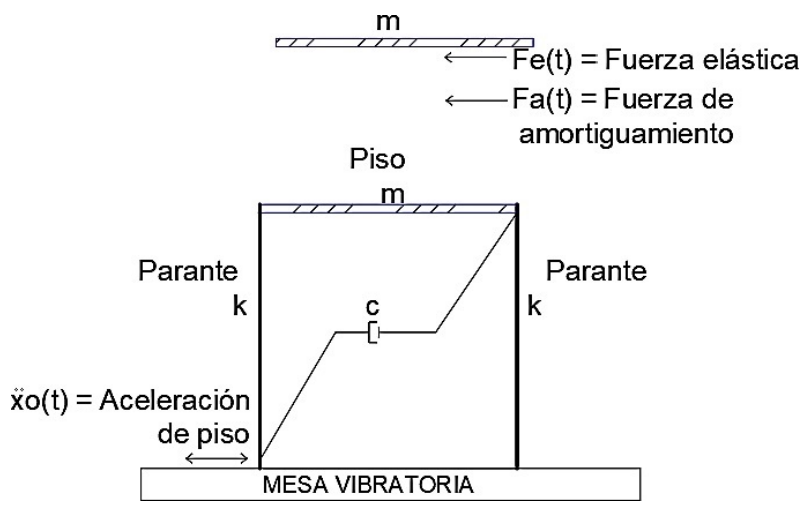

Figura 5. Fuerzas actuantes 


\subsection{Método de Newmark}

Es un método muy versátil, para el cálculo de la respuesta un sistema dinámico. En 1959, N. M. Newmark desarrolló una familia de métodos dependiendo de la ley de variación entre instantes consecutivos de tiempo [11]; el que se utilizó es el de aceleración lineal como se muestra en la Figura 6.

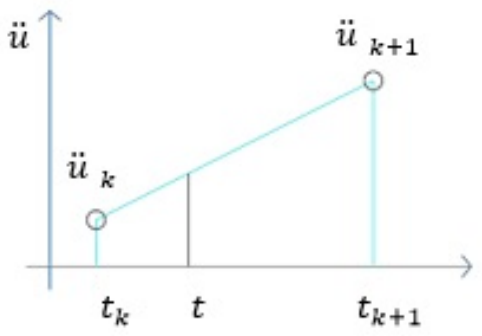

Figura 6. Aceleración lineal

La representación matricial del método aplicado para la obtención de las respuestas de aceleración, velocidad y posición en el tiempo para un sistema de un grado de libertad, se muestra en la Ecuación (10), donde la respuesta en instante $k+1$, solo depende de la respuesta del instante $k$ [11].

$$
\left(\begin{array}{l}
u_{k+1} \\
\dot{u}_{k+1} \\
\ddot{u}_{k+1}
\end{array}\right)=[A] \times\left(\begin{array}{l}
u_{k} \\
\dot{u}_{k} \\
\ddot{u}_{k}
\end{array}\right)+[B] \times \ddot{x} o_{k+1}
$$

Donde $[A],[B]$ son matrices de constantes que dependen de la frecuencia, amortiguamiento, rigidez e intervalo de tiempo y $\ddot{x} o_{k+1}$ es aceleración de piso en el instante $k+1$ [12] y [18].

\subsubsection{Estabilidad del método}

Existen dos tipos de métodos dependiendo de la estabilidad de los mismos: condicionalmente estables e incondicionalmente estables [11].

Los procedimientos condicionalmente estables dependen del paso del tiempo y los procedimientos incondicionalmente estables son independientes del paso del tiempo [11]

El método de Newmark con base en la aceleración lineal es condicionalmente estable y debe cumplir con la Ecuación (11), para poder ser aplicado.

$$
\frac{\Delta t}{T}<0,551
$$

Donde $\Delta t$ es el intervalo de tiempo y $T$ es el periodo del sistema.

\subsection{Análisis modal}

Permite calcular la respuesta de la estructura con base en los modos de vibración. Para ello es importante definir sus propiedades dinámicas: frecuencia, amortiguamiento y formas modales para cada modo [19].

Este análisis es muy útil porque permite descomponer las respuestas de una estructura en modelos de un solo grado de libertad y al combinarlas obtener la respuesta del sistema MDOF.

\subsubsection{Frecuencias y modos de vibración}

En el sistema amortiguado de múltiples grados de libertad, se debe establecer las frecuencias naturales y las formas modales considerándolo como si se tratase de un sistema sujeto a vibración libre y sin amortiguamiento, por lo cual en la Ecuación (9), los términos de la aceleración de piso y amortiguamiento se anulan, y se obtiene la Ecuación (12).

$$
[M] \times \ddot{u}+[K] \times\{u\}=0
$$

Resolviendo esta ecuación diferencial se llega a la Ecuación (13), la cual es la base para la resolución del problema de valores y vectores propios [11].

$$
\left[[K]-[M] \times\left\{\omega^{2}\right\}\right] \times\{\phi\}=0
$$

La Ecuación (13) corresponde a un sistema de ecuaciones simultáneas homogéneas, por definición solo tiene solución no trivial, por tanto, el determinante de la matriz de coeficientes es cero, como se indica en la Ecuación (14) [20].

$$
\left|[K]-[M] \times\left\{\omega^{2}\right\}\right|=0
$$

$\mathrm{Al}$ expandir el determinante se obtiene un polinomio de orden $2 n$ (n: número de modos de vibración), donde $\omega^{2}$ es la variable. Esta ecuación se la conoce como «ecuación de frecuencias». Las soluciones se denominan valores propios y las raíces cuadradas de estas, corresponde a las frecuencias naturales del sistema $\omega[21]$.

Para determinar los vectores propios o modos de vibración $\phi$, se sustituye las frecuencias naturales $\omega$ en la Ecuación (13) y se resuelve.

\subsubsection{Ortogonalidad de los modos}

El análisis anterior permite demostrar que los modos de vibración de diferentes frecuencias, cumplen la condición de ortogonalidad, que se muestra a continuación (15) [11].

$$
\begin{array}{r}
\{\phi i\}^{T} \times[M] \times\{\phi j\}=0 \\
\{\phi i\}^{T} \times[K] \times\{\phi j\}=0 \\
i \neq j \text { Donde } i, j: \text { modos de vibración }
\end{array}
$$

Donde $\phi i$ y $\phi j$ son formas modales para los modos $i$ y $j,[M]$ matriz de masa y $[K]$ matriz de rigidez. 
A partir de ello, se obtiene que el sistema se puede resolver para cada modo de vibración como un sistema separado sin influencia de un modo con respecto al otro, y la respuesta del sistema completo se define con base en la Ecuación (16).

$\{x\}=\{\phi 1\} \times q 1(t)+\{\phi 2\} \times q 2(t)+\cdots\{\phi n\} \times q n(t)$

$\{\dot{x}\}=\{\phi 1\} \times \dot{q} 1(t)+\{\phi 2\} \times \dot{q} 2(t)+\cdots\{\phi n\} \times \dot{q n}(t)$

$\{\ddot{x}\}=\{\phi 1\} \times \ddot{q} 1(t)+\{\phi 2\} \times \ddot{q} 2(t)+\cdots\{\phi n\} \times \ddot{q} n(t)$

Donde $\phi_{i}$ forma modal, y $q, \dot{q}$ y $\ddot{q}$ son las coordenadas modales de posición, velocidad y aceleración, en el modo i-simo.

Reemplazando la respuesta $\{x\},\{\dot{x}\}$ y $\ddot{x}$ con base en la suma de los modos y premultiplicando por la matriz de formas modales transpuestas, se obtiene la ecuación (17).

$$
\begin{array}{r}
{[\Phi]^{T}[M][\Phi] \times \ddot{q}_{n}+[\Phi]^{T}[C] \times[\Phi] \times \dot{q}_{n}+} \\
{[\Phi]^{T} \times[K] \times[\Phi] \times q_{n}=-[\Phi]^{T} \times[M] \times\{\iota\} \times \ddot{x} o}
\end{array}
$$

Basándose en la Ecuación (17) y la propiedad de ortogonalidad, se puede definir la Ecuación (18) para cada modo de vibración.

$$
\begin{array}{r}
{\left[\phi_{n}\right]^{T}[M]\left[\phi_{n}\right] \times \ddot{q}_{n}+\left[\phi_{n}\right]^{T}[C] \times\left[\phi_{n}\right] \times \dot{q}_{n}+} \\
{\left[\phi_{n}\right]^{T} \times[K] \times\left[\phi_{n}\right] \times q=-\left[\phi_{n}\right]^{T} \times[M] \times\{\iota\} \times \ddot{x} O}
\end{array}
$$

A partir de la Ecuación (18) y con ayuda del método Newmark de aceleración lineal, se obtiene respuestas dinámicas relativas (posición, velocidad y aceleración) en cada instante de tiempo para cada coordenada modal.

\subsection{Diseño del programa}

\subsubsection{Lenguaje del programa MatLab}

El programa ATH Dynamic responses ha sido desarrollado en el software Matlab, el cual es un lenguaje de programación con un entorno de trabajo amigable. Permite trabajar en modo consola (tan solo se presenta resultados con base en expresiones planteadas) y en modo rutina (programas cuyos comandos están codificados y permiten realizar programas y grabarlos) [7] y [22].

La característica más importante de Matlab es que permite un manejo directo de vectores y matrices, además que la codificación no es compleja [7].

El proyecto se realizó en modo rutina, debido a que se ejecutó un programa con comandos codificados y se creó un GUIDE, que permitió mejorar la interfaz gráfica para el usuario.

\subsubsection{Programa ATH Dynamic responses}

El programa ATH Dynamic responses se basa en el método numérico de Newmark considerando aceleración lineal para el cálculo de la respuesta de los sistemas de 1 GDL en el análisis modal.

Dentro del programa se deben ingresar las características geométricas, la rigidez de los parantes y la masa considerada como concentrada en cada piso. Con base en esto se obtienen respuestas dinámicas relativas (posición, velocidad y aceleración) y propiedades modales del modelo físico (frecuencia, modos y porcentaje de masa participativa).

Para comprender la funcionabilidad del programa, en la Figura 7, se indica el diagrama de flujo del mismo. 


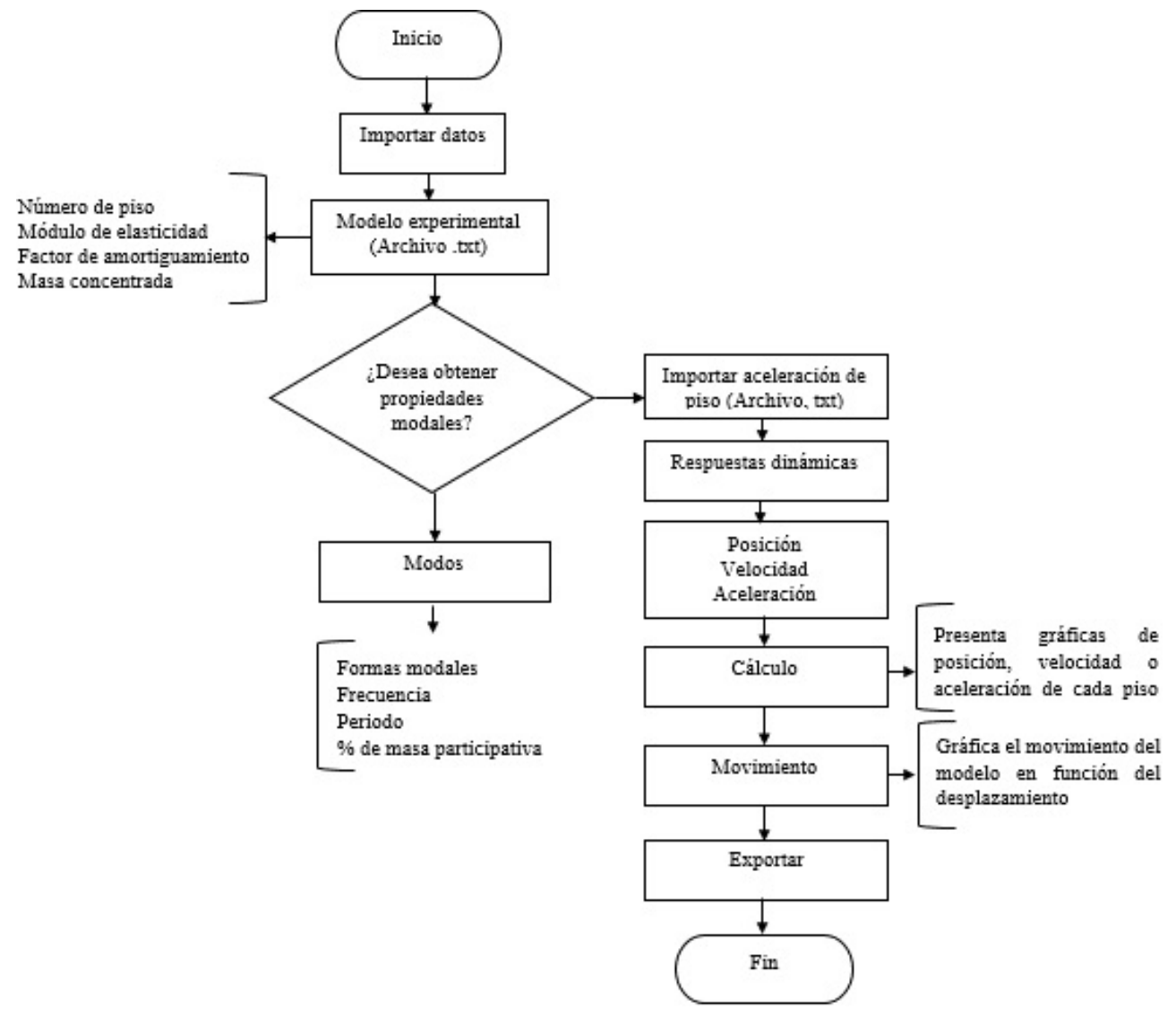

Figura 7. Diagrama de flujo del programa ATH Dynamic responses

\subsection{Procesamiento de datos}

Los datos obtenidos por los acelerómetros necesitan ser corregidos por línea base y filtrado de frecuencias no deseadas. Para lo cual se utilizó el filtro butterworth [23], pasa bajo una frecuencia de corte de $16 \mathrm{~Hz}$. Con este objetivo se ha utilizado el programa AB signal [24].

\subsection{Instrumentación}

En general, la instrumentación para medir la respuesta dinámica de una estructura, consta de la instalación de sensores que registren respuestas dinámicas (velocidad, aceleración y desplazamiento) [25].

La finalidad de la instrumentación es registrar la respuesta frente al desplazamiento, movimientos internos, sismos y observar el comportamiento de estructuras o modelos [20].

La adquisición de datos del proyecto se efectuó mediante acelerómetros de PCB Piezotronics [25], los cuales tienen frecuencias y sensibilidades que se muestran en la Tabla 1.
Tabla 1. Propiedades de los acelerómetros

\begin{tabular}{ccc}
\hline Tipo & Frecuencia & Sensibilidad \\
\hline $\begin{array}{c}\text { Acelerómetro } \\
\text { normal }\end{array}$ & $2 \mathrm{~Hz}-5 \mathrm{kHz}$ & $100 \mathrm{~m} \mathrm{~V} / \mathrm{g}$ \\
$\begin{array}{c}\text { Acelerómetro } \\
\text { normal }\end{array}$ & $1 \mathrm{~Hz}-5 \mathrm{kHz}$ & $100 \mathrm{~m} \mathrm{~V} / \mathrm{g}$ \\
$\begin{array}{c}\text { Acelerómetro } \\
\text { miniatura }\end{array}$ & $1 \mathrm{~Hz}-4 \mathrm{kHz}$ & $100 \mathrm{~m} \mathrm{~V} / \mathrm{g}$ \\
\hline
\end{tabular}

\subsection{Mesa vibratoria (Shake Table II)}

El ensayo en una mesa vibratoria es la forma más directa para simular el comportamiento dinámico de las estructuras. Los modelos estarán limitados a no ser muy pesados, de dimensiones escaladas y no muy rígidos [26].

Shake Table II se desarrolló originalmente por la University Consortium on Instructional Shake Tables (UCIST) [6]. Es un dispositivo mecánico, que consta de una placa superior de $45,7 \times 45,7 \mathrm{~cm}^{2}$ donde se ancla el modelo, una placa inferior de 60,9 × 45,7 $\mathrm{cm}^{2}$ y un motor DC con una potencia de $400 \mathrm{~W}$. La mesa soporta una carga de 7,5 kg a una aceleración de 
2,5 g y permite movimientos con un recorrido de hasta $\pm 7,62 \mathrm{~cm}[6]$.

\subsection{Materiales}

El equipo utilizado para la realización del proyecto está conformado por una mesa vibratoria Shake Table II [6] y acelerómetros de PCB Piezotronic [25], descritos en la sección 2.11. y 2.12 .

Los modelos experimentales están conformados por acrílicos y acero inoxidable. En las Tablas 2 y 3 se especifican las características y dimensiones de los materiales.

Tabla 2. Especificaciones de los materiales de los parantes y los pisos

\begin{tabular}{cccccc}
\hline Material & $\begin{array}{c}\text { Espesor } \\
(\mathbf{m})\end{array}$ & $\begin{array}{c}\text { Ancho } \\
(\mathbf{m})\end{array}$ & $\begin{array}{c}\text { Longitud } \\
(\mathbf{m})\end{array}$ & $\begin{array}{c}\mathbf{I} \\
\left(\mathbf{m}^{4}\right)\end{array}$ & $\begin{array}{c}\mathbf{E} \\
\left(\mathbf{k N} / \mathbf{m}^{2}\right)\end{array}$ \\
\hline $\begin{array}{c}\text { Parante } \\
\text { acero } \\
\text { inoxidable }\end{array}$ & $7 \mathrm{E}^{-4}$ & 0,06 & 0,3 & $1,715 \mathrm{E}^{-12}$ & $1,80 \mathrm{E}^{+11}$ \\
\hline $\begin{array}{c}\text { Piso } \\
\text { acrílico }\end{array}$ & 0,01 & 0,06 & 0,31 & & \\
\hline
\end{tabular}

Tabla 3. Masa de los parantes y pisos

\begin{tabular}{ccc}
\hline & Material & Masa $(\mathrm{g})$ \\
\hline Pisos & Acrílico & $232-234$ \\
\hline $\begin{array}{c}\text { Parante } \\
\text { modelo 1 }\end{array}$ & $\begin{array}{c}\text { Acero } \\
\text { inoxidable }\end{array}$ & 102 \\
\hline $\begin{array}{c}\text { Parante } \\
\text { modelo 2 }\end{array}$ & $\begin{array}{c}\text { Acero } \\
\text { inoxidable }\end{array}$ & 204 \\
\hline $\begin{array}{c}\text { Parante } \\
\text { modelo 3 }\end{array}$ & $\begin{array}{c}\text { Acero } \\
\text { inoxidable }\end{array}$ & 299 \\
\hline
\end{tabular}

En la Figura 8 se presenta los gráficos de los modelos experimentales que se construyeron. En la Figura 9 se indica las dimensiones del modelo $3 \mathrm{y}$, además, cómo se realizó la concentración de la masa para el modelo teórico, con base en el modelo experimental y en la Figura 10 se puede observar una imagen del modelo real.

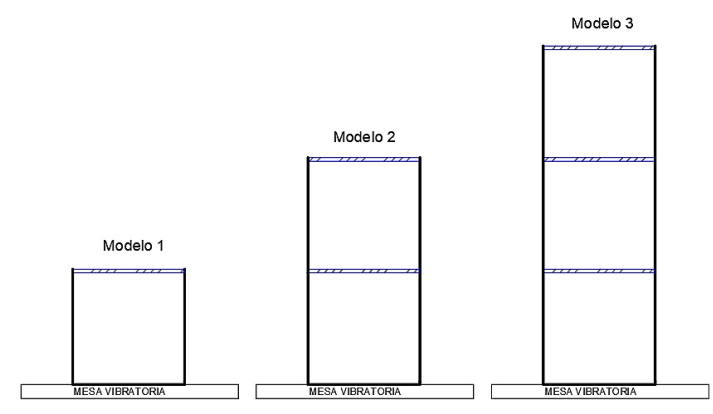

Figura 8. Modelo de uno, dos y tres pisos

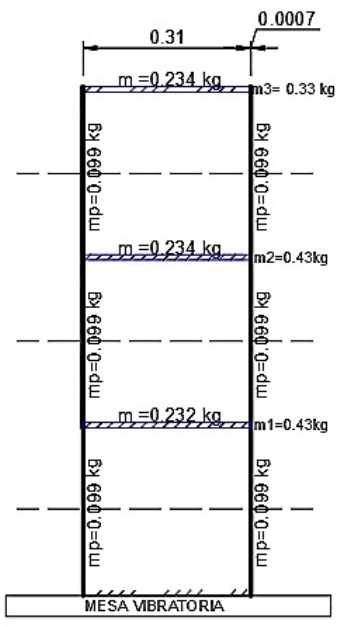

Vista frontal

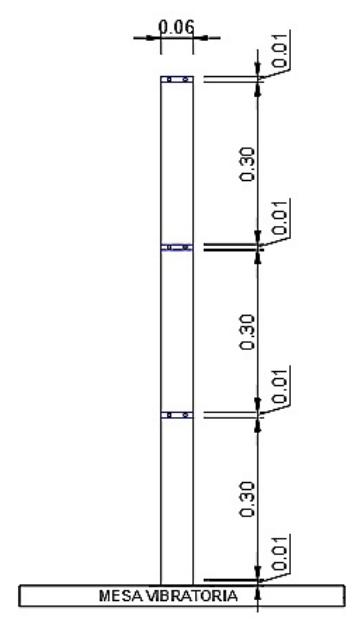

Vista lateral
Figura 9. Dimensiones del modelo de 3 grados de libertad y concentración de masa

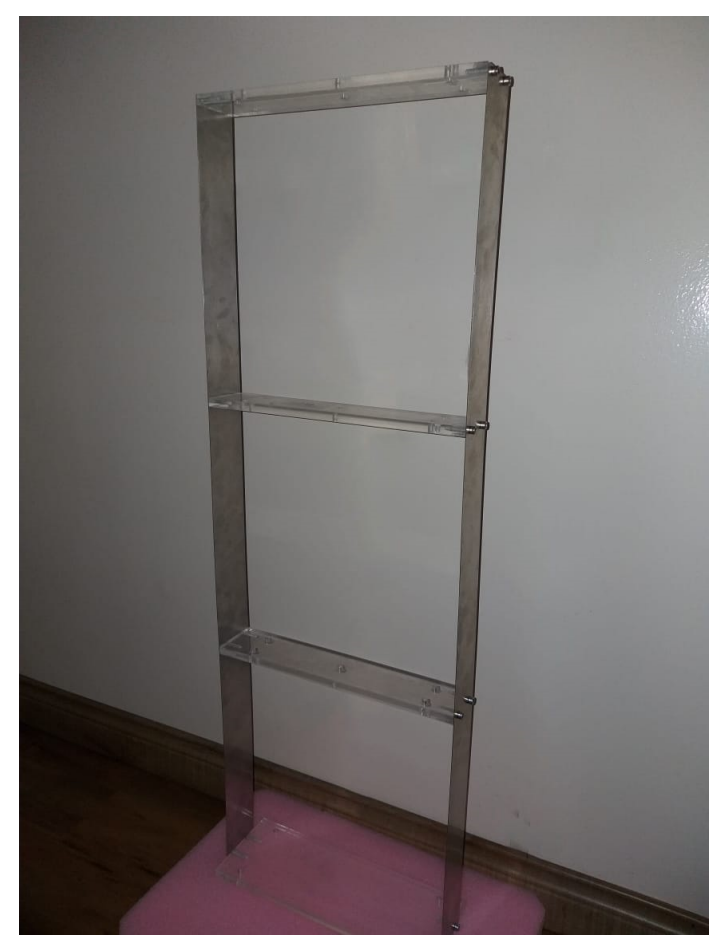

Figura 10. Modelo experimental del modelo de 3 grados de libertad

\section{Resultados y discusión}

Los resultados muestran frecuencias, factores de amortiguamiento, aceleraciones absolutas de los modelos experimentales y su comparación con los resultados teóricos proporcionados por el programa $A T H D y$ namic responses. 
3.1. Experimento 1. Obtención de frecuencias y factores de amortiguamiento de cada modelo

Las frecuencias se obtuvieron de manera teórica mediante el programa ATH Dynamic responses y verificadas con el software SAP2000 [27]. Para la obtención de la frecuencia experimental y el factor de amortiguamiento, se realizó un barrido de frecuencias con la Shake Table II.

Se efectuó el proceso de corrección de línea base y filtrado, como se explicó en la sección 2.10. A partir de ello se utilizó la transformada rápida de Fourier disponible en Matlab [23] y se obtuvo gráficas de transmisibilidad vs. frecuencia como se muestran en las Figuras 11, 12 y 13, donde a la abscisa de cada pico del gráfico le corresponde la frecuencia de cada modo y, además, permite obtener el factor de amortiguamiento aplicando la Ecuación (8). El error porcentual en la frecuencia analítica que se obtuvo es con respecto a la aceleración experimental.

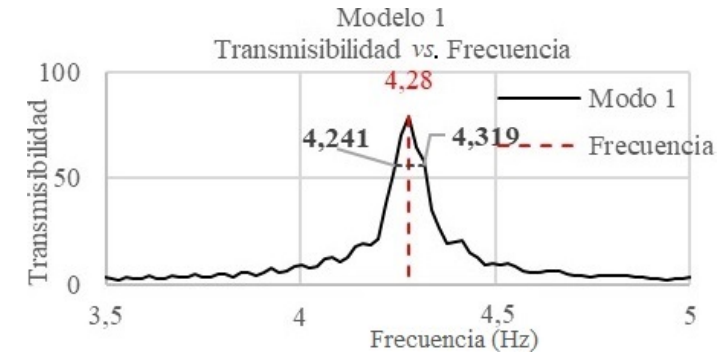

Figura 11. Frecuencia y ancho de banda del modelo 1

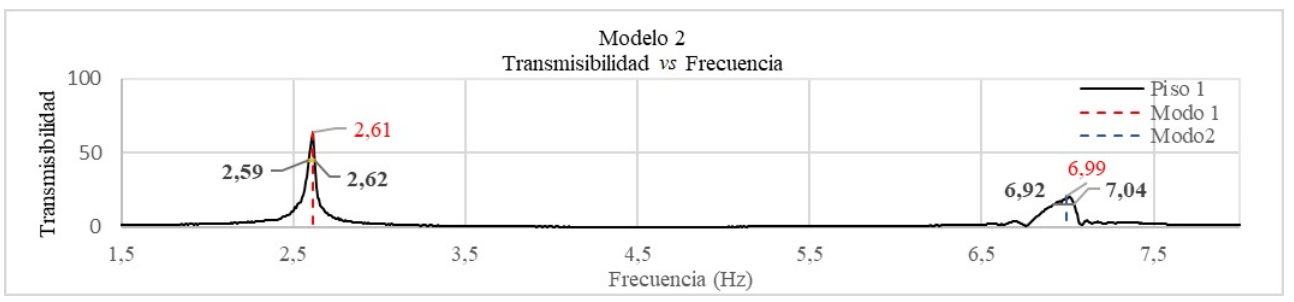

(a)

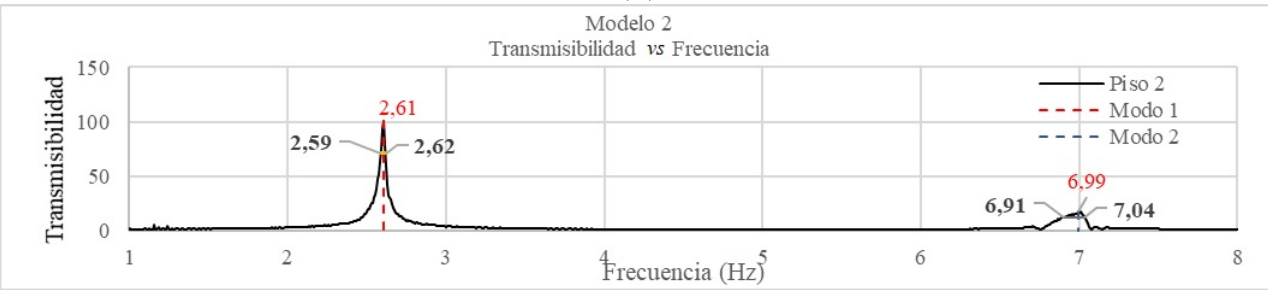

(b)

Figura 12. Frecuencia y ancho de banda del modelo 2 (a) piso 1 y (b) piso 2

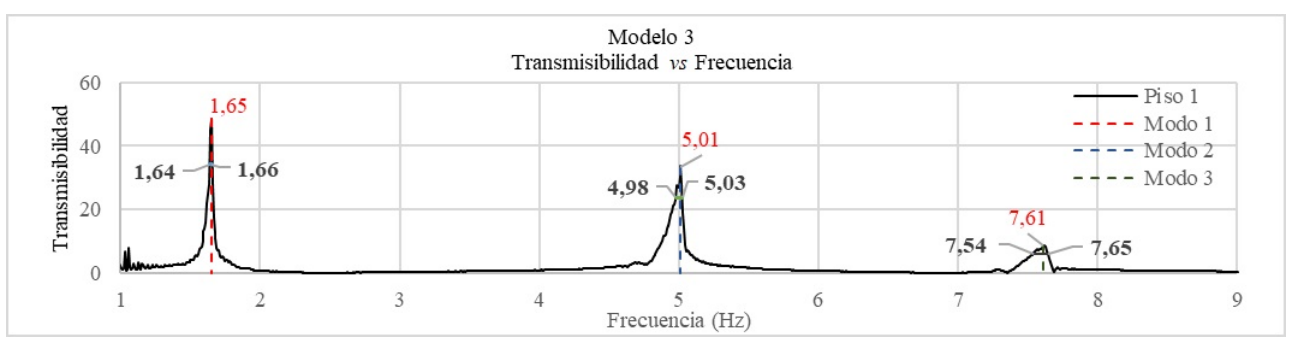

(a)

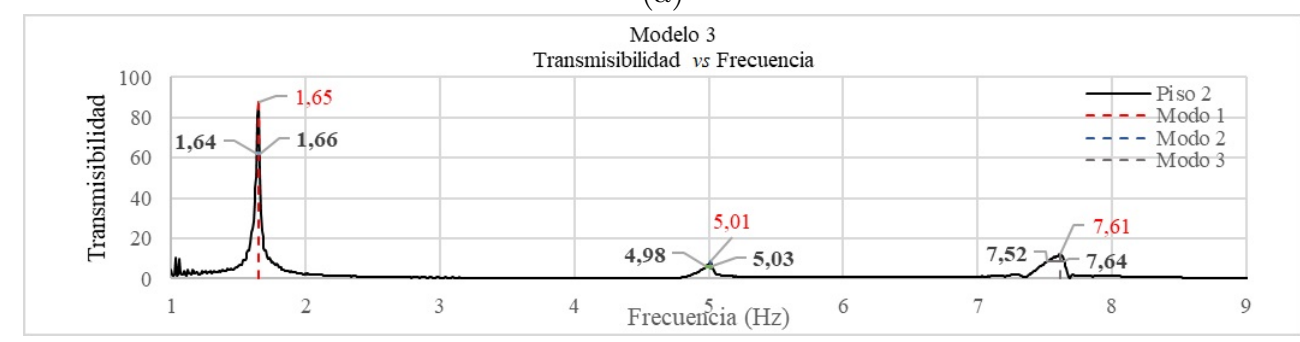

(b) 


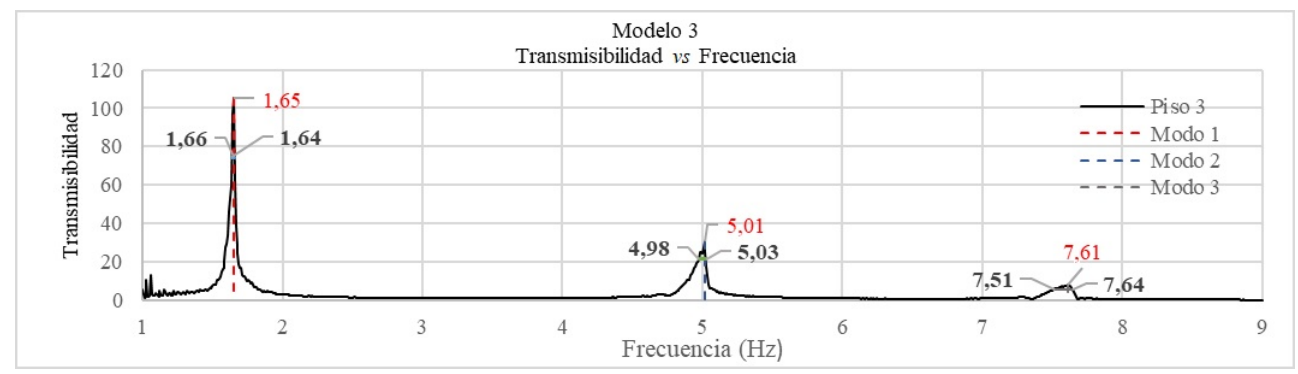

(c)

Figura 13. Frecuencia y ancho de banda del modelo 3 (a) piso 1, (b) piso 2 y (c) piso 3

\subsubsection{Frecuencias y factor de amortiguamiento del modelo 1}

En la Figura 11 se muestran las gráficas que permitieron obtener la frecuencia teórica y el factor de amortiguamiento, y en la Tabla 4 se tabulan estos valores.

El peak de la Figura 11 muestra que la frecuencia experimental para el modelo 1 es $4,28 \mathrm{~Hz}$.

Tabla 4. Frecuencia y amortiguamiento modelo 1

\begin{tabular}{ccccc}
\hline & $\begin{array}{c}\text { ATH } \\
\text { Dynamic } \\
\text { responses }\end{array}$ & Exp. & & Amortiguamiento \\
\hline Modo & $\mathbf{f ~ ( \mathbf { H z } )}$ & $\mathbf{f ~ ( H z )}$ & \% error & $\xi(\%)$ \\
\hline 1 & 4,58 & 4,28 & 6,55 & 1,44 \\
\hline
\end{tabular}

\subsubsection{Frecuencias $y$ factor de amortiguamiento del modelo 2}

En las Figuras 12 (a) y (b) se muestran las gráficas que permitieron obtener las frecuencias y los factores de amortiguamiento para los dos modos y en la Tabla 5 se presentan los resultados.

Los picos de las Figuras 12 (a) y (b) son la frecuencia experimental, para el modo 1 es $2,61 \mathrm{~Hz}$ y para el modo 2 es $6,99 \mathrm{~Hz}$.

Tabla 5. Frecuencia y amortiguamiento modelo 2

\begin{tabular}{ccccc}
\hline & $\begin{array}{c}\text { ATH } \\
\text { Dynamic } \\
\text { responses }\end{array}$ & Exp. & & Amortiguamiento \\
\hline Modo & $\mathbf{f ~ ( H z )}$ & $\mathbf{f ~ ( H z )}$ & \% error & $\xi(\%)$ \\
\hline 1 & 2,61 & 2,60 & 0,38 & 0,57 \\
2 & 6,53 & 6,99 & 7,07 & 0,85 \\
\hline
\end{tabular}

\subsubsection{Frecuencias y factor de amortiguamiento del modelo 3}

En la Figura 13 (a), (b) y (c) se muestran las gráficas que permitieron obtener las frecuencias, los factores de amortiguamiento para los 3 modos y en la Tabla 6 , se presentan los resultados.

Los picos de las Figuras 13 (a), (b) y (c), son las frecuencias experimentales, para el modo 1 es
$1,65 \mathrm{~Hz}$, para el modo 2 es $5,01 \mathrm{~Hz}$ y para el modo 3 es $7,61 \mathrm{~Hz}$.

Tabla 6. Frecuencia y amortiguamiento modelo 3

\begin{tabular}{ccccc}
\hline & $\begin{array}{c}\text { ATH } \\
\text { Dynamic } \\
\text { responses }\end{array}$ & Exp. & & Amortiguamiento \\
\hline Modo & $\mathbf{f ~ ( H z )}$ & $\mathbf{f ~ ( H z )}$ & \% error & $\xi(\%)$ \\
\hline 1 & 1,91 & 1,65 & 13,61 & 0,55 \\
2 & 5,25 & 5,01 & 4,57 & 0,49 \\
3 & 7,36 & 7,6 & 3,26 & 0,85 \\
\hline
\end{tabular}

\subsection{Experimento 2. Respuesta dinámica del modelo a aceleración de piso}

La respuesta dinámica se obtuvo al someter a los modelos experimentales a un registro sísmico escalado. El registro sísmico que se utilizó fue El Centro y su escalamiento se realizó mediante el software de la Shake Table II; el escalado del registro fue sobre la base de un desplazamiento máximo de $4 \mathrm{~cm}$.

Los resultados que se presentan a continuación son de aceleraciones absolutas, debido a que estas son las que proporcionan directamente los acelerómetros y se compararon con las aceleraciones absolutas obtenidas del programa ATH Dynamic response, con base en la Ecuación (19):

$$
\ddot{x}=\ddot{u}+\ddot{x}_{o}
$$

Donde $\ddot{x}$ es aceleración absoluta, $\ddot{u}$ es aceleración relativa y $\ddot{x}_{o}$ aceleración de base.

\subsubsection{Modelo 1 registro de aceleración en base: sismo El Centro}

En la Figura 14, se muestra la aceleración experimental y la aceleración teórica del modelo de 1 piso, y en la Tabla 7, las aceleraciones máximas teóricas y experimentales.

Tabla 7. Aceleraciones máximas modelo 1

\begin{tabular}{|c|c|c|}
\hline \multicolumn{3}{|c|}{ Aceleración $\left(\mathrm{m} / \mathrm{s}^{2}\right)$ Piso 1} \\
\hline Teórico & Experimental & $\%$ error \\
\hline 8,707 & 7,657 & 13,711 \\
\hline
\end{tabular}




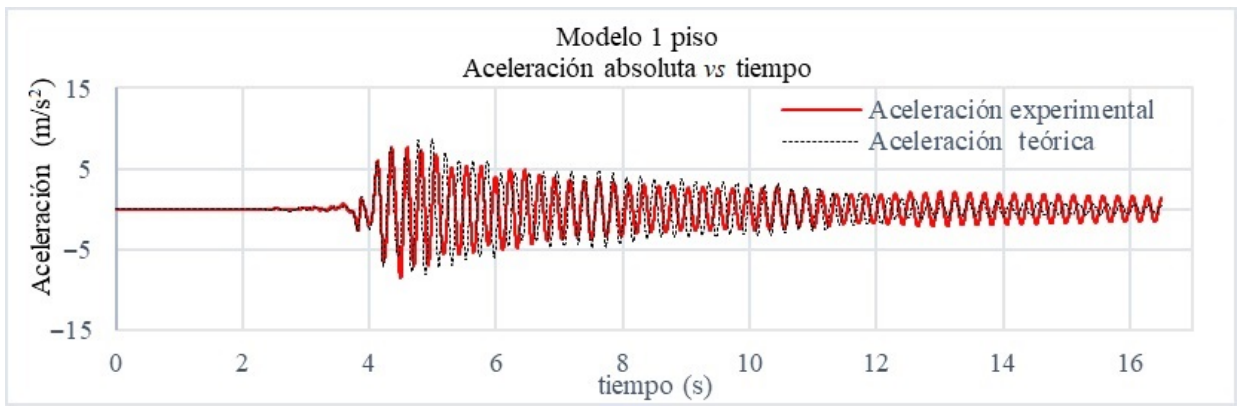

Figura 14. Aceleración absoluta experimental y teórica del modelo 1, con aceleración en la base del sismo El Centro

\subsubsection{Modelo 2 aceleración base: sismo El Centro}

En las Figuras 15 (a) y (b) se muestra la aceleración experimental y la aceleración teórica del modelo 2 y en la Tabla 8 las aceleraciones máximas teóricas y experimentales.
Tabla 8. Aceleraciones máximas modelo 2

\begin{tabular}{lccc}
\hline \multicolumn{4}{c}{ Aceleración $\left(\mathrm{m} / \mathrm{s}^{2}\right)$} \\
\hline & Teórico & Experimental & $\%$ error \\
\hline Piso 1 & 7,585 & 8,678 & 12,586 \\
Piso 2 & 8,989 & 9,021 & 0,353 \\
\hline
\end{tabular}

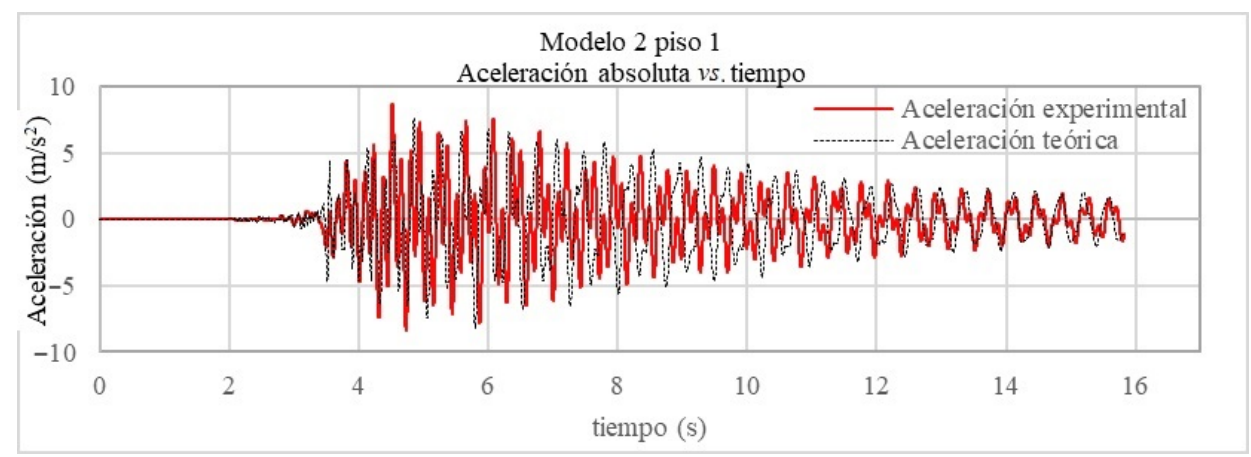

(a)

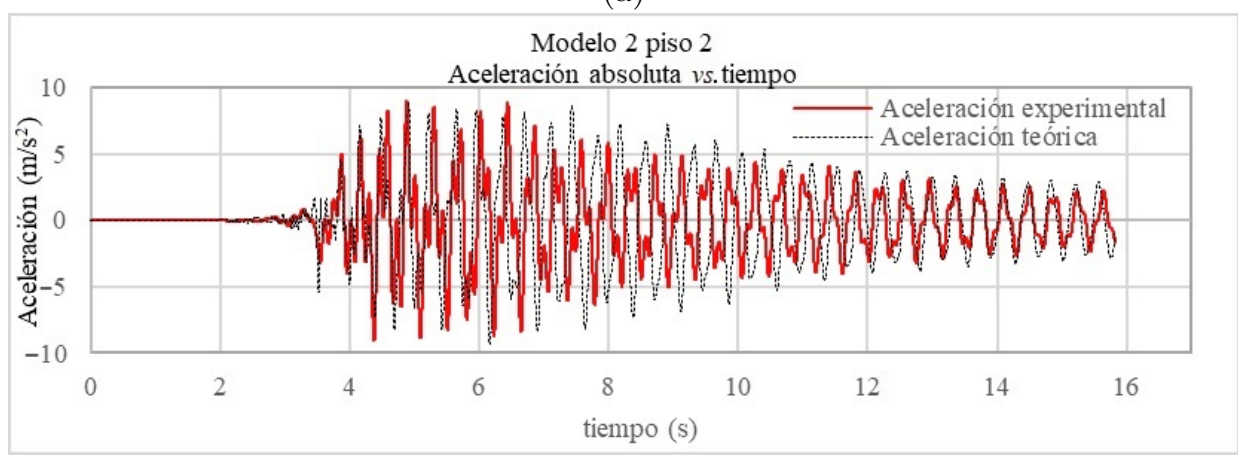

(b)

Figura 15. Aceleración absoluta experimental y teórica del modelo 2, con aceleración en la base del sismo El Centro (a) piso 1 (b) piso 2

\subsubsection{Modelo 3 aceleración base: sismo El Centro}

En las Figuras 16 (a), (b) y (c) se muestra la aceleración experimental y la aceleración teórica del modelo 3 y en la Tabla 9 las aceleraciones máximas teóricas y experimentales.
Tabla 9. Aceleraciones máximas modelo 2

\begin{tabular}{cccc}
\hline \multicolumn{4}{c}{ Aceleración $\left(\mathrm{m} / \mathrm{s}^{2}\right)$} \\
\hline & Teórico & Experimental & $\%$ error \\
\hline Piso 1 & 5,225 & 5,033 & 3,824 \\
Piso 2 & 5,297 & 5,698 & 7,043 \\
Piso 3 & 5,967 & 5,845 & 2,095 \\
\hline
\end{tabular}




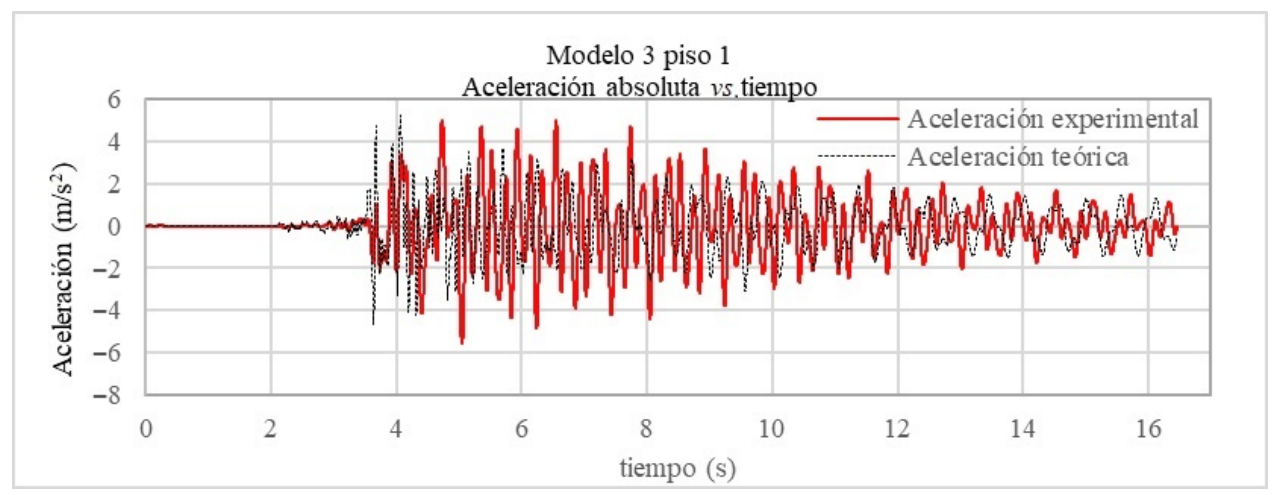

(a)

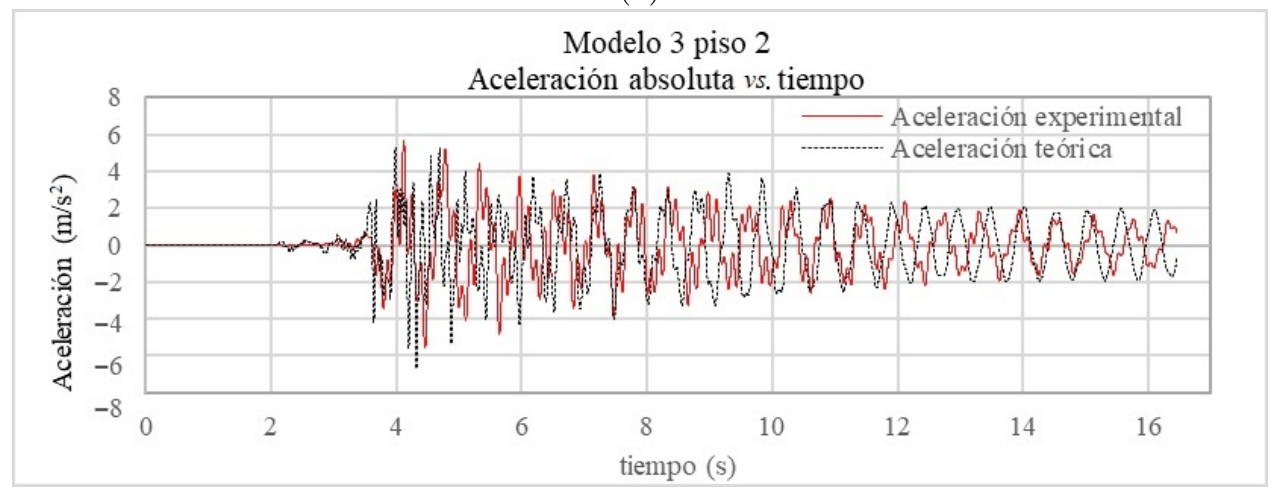

(b)

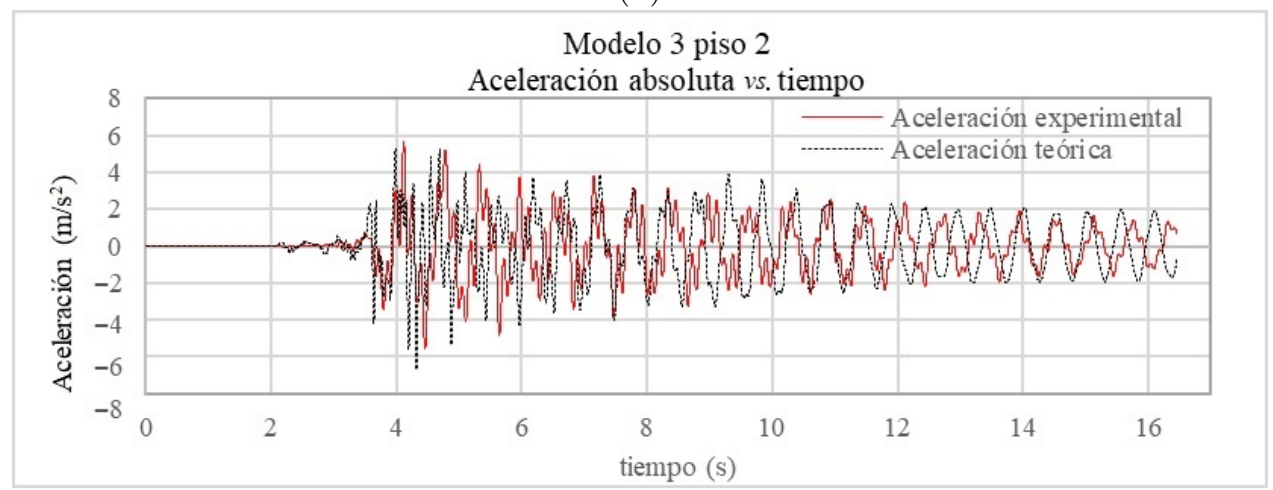

(c)

Figura 16. Aceleración absoluta experimental y teórica del modelo 3, con aceleración en la base del sismo El Centro (a) piso 1 (b)piso 2 (c) piso 3

Al comparar gráficamente los modelos, se observó que la aceleración experimental tiene mayor amplitud en la mayoría de los casos, sin embargo, de manera general las gráficas experimental y teórica son semejantes en su forma.

\section{Conclusiones}

Con base en los resultados de la experimentación y los resultados teóricos de los modelos, se puede argumentar que el método para la obtención de frecuencias con el uso de la transformada de Fourier, es adecuado debido a que los resultados son semejantes entre frecuencias experimentales y teóricas.
El factor de amortiguamiento obtenido mediante el ancho de banda es coherente en cuanto a los valores obtenidos por piso, debido a que cada piso proporcionó valores similares de factores de amortiguamientos por modo y dichos factores fueron útiles para la modelación teórica, y así poder obtener la matriz de amortiguamiento y las respuestas dinámicas.

La modelación experimental del modelo, nos ayudó a observar de manera real cómo se comporta un modelo ante la aplicación de una aceleración sinusoidal o aceleración de un sismo escalado, y permitió verificar los valores obtenidos analíticamente respecto de los experimentales. 
Como trabajo futuro se puede implementar la obtención de propiedades dinámicas de sistemas tridimensionales que presenten irregularidad en planta y elevación.

Se puede observar un desfase entre las respuestas experimentales y las teóricas en cuanto a las aceleraciones absolutas, a causa de la consideración del tiempo 0 , en que los modelos experimentales estaban en estado de reposo.

\section{Referencias}

[1] INEC, Reconstruyendo las cifras luego del sismo. Memorias. Instituto Nacional de Estadísticas y Censos. Ecuador, 2017. [Online]. Available: https://bit.ly/3gEu4Al

[2] Instituto Geofísico. (2020) Cuatro años después del terremoto de Pedernales: un testimonio sobre el peligro sísmico en el Ecuador. [Online]. Available: https://bit.ly/3dW198V

[3] MIDUVI, Norma ecuatoriana de la construcción. NEC-SE-DS. Cargas Sísmicas. Diseño Sismoresistente. Ministerio de Desarrollo Urbano y Vivienda. Ecuador, 2014. [Online]. Available: https://bit.ly/3xpr2FY

[4] D. Gutiérrez Calzada, Sistema de múltiples grados de libertad. Análisis modal espectral. Universidad Autónoma del Estado de México. México, 2018. [Online]. Available: https://bit.ly/3dSSQe2

[5] D. Henao Ángel, "Identificación de las propiedades dinámicas de una estructura sometida a vibración ambiental empleando análisis espectral," Master's thesis, 2013. [Online]. Available: https://bit.ly/3sQd7Fu

[6] QUANSER, "SHAKE TABLE II bench-scale single-axis motion simulator," QUANSER INNOVATE EDUCATE, Tech. Rep., 2020. [Online]. Available: https://bit.ly/3tWivbj

[7] J. E. Hurtado Gómez, Introducción de la dinámica de estructuras. Universidad Nacional de Colombia, sede Manizales, 2000. [Online]. Available: https://bit.ly/3ezgOKC

[8] B. A. Guaygua Quillupangui, V. N. Colcha Guachamín, and E. L. Tibán Guacollante, "Estudio comparativo del comportamiento dinámico de modelos estructurales teóricos y modelos estructurales experimentales," 2018. [Online]. Available: https://bit.ly/3gJDR8i

[9] P. X. Villalba Nieto, A. I. Cepeda Aveiga, and N. A. Hipocuro Simbaña, "Análisis de las frecuencias fundamentales de modelos estructurales con excitaciones sísmicas," 2019. [Online]. Available: https://bit.ly/3dXrBio

[10] L. W. Morales Gubio, P. E. Chimarro Quishpe, and M. G. Coronel Armas, "Análisis de una estructura de un edificio de 9 piso a escala en Quito, sometido a cargas sísmicas en la mesa de vibración," 2018. [Online]. Available: https://bit.ly/32Ts5A4

[11] A. K. Chopra, Dinámica de Estructuras. Pearson Educación de México, 2014. [Online]. Available: https://bit.ly/3dROhkd

[12] Y. Bai and Z.-D. Xu, Structural Dynmics for Structural Engineers. John Wiley \& Sons, Inc., 2019. [Online]. Available: https://bit.ly/3tYARsf

[13] R. R. Craig and A. J. Kurdila, Fundamentals of Structural Dynamics. John Wiley \& Sons, Inc, 2006. [Online]. Available: https://bit.ly/2S6J6EK

[14] O. Möller, M. Rubinstein, and J. P. Ascheri, "Análisis del amortiguamiento proporcional a la rigidez tangente en sistemas dinámicos no lineales," Asociación Argentina de Mecánica Computacional, vol. XXX, no. 14, pp. 1277-1293, 2011. [Online]. Available: https://bit.ly/3evo9L8

[15] D. J. Inman, Vibration with Control. John Wiley \& Sons, Inc, 2017. [Online]. Available: https://bit.ly/3vidqKE

[16] R. Boroschek and F. Hernández, "Corrección de sobreestimación del amortiguamiento en el método de ancho de banda del espectro de potencia," in X Chilean Conference of seismology and earthquake engineering. ACHISINA, 2010. [Online]. Available: https://bit.ly/32Uy2wp

[17] M. Paz, Dinámica estructural teoría y cálculo. Editorial Reverté, 1992. [Online]. Available: https://bit.ly/32Pw94l

[18] P. L. Sierra, O. Möller, J. P. Ascheri, and M. Poliotti, "Dinámica estructural, comparación y análisis de la propagación de vibraciones en estructuras," in XXIII Congreso de Métodos Numéricos y sus Aplicaciones (ENIEF 2017), 2017. [Online]. Available: https://bit.ly/32Wc780

[19] M. Rodríguez, "Análisis modal operacional: Teoría y práctica," 2005. [Online]. Available: https://bit.ly/3gPU5wm

[20] H. Garzón Molano, "Instrumentación geotécnica. aplicación y soporte para la toma de decisiones," 2018. [Online]. Available: https://bit.ly/3aF31Ru

[21] L. E. García Reyes, Dinámica estructural aplicada al diseño sísmico. Universidad de los Andes. Bogotá - Colombia, 1998. 
[22] R. Aguiar Falconi, Dinámica de estructuras con MatLab. Centro de Investigación Científica, CEINCI - Universidad de las Fuerzas Armada, ESPE, 2006. [Online]. Available: https://bit.ly/3dU0dlC

[23] MathWorks. (2021) Matlab para inteligencia artificial. 1994-2021 The MathWorks, Inc. [Online]. Available: https://bit.ly/2S9ay4I

[24] B. D. Erazo Silva and P. A. Vargas Yépez, "Desarrollo de un software para procesamiento y corrección de registros, y generación de espectros de respuesta sísmica," 2020. [Online]. Available: https://bit.ly/3u0ZAfB
[25] DataLights. (2020) Soluciones de control e iluminación. Datalights, Cia. Ltda. [Online]. Available: https://bit.ly/3aH2T3U

[26] L. Bustos, F. Zabala, J. Santalucía, and A. Masanet, "Estudio del comportamiento dinámico de un modelo de mampostería encadenada mediante un ensayo en mesa vibratoria," in JUBILEO, XXIX Jornadas Sudamericanas de Ingeniería Estructural, Argentina, 2020. [Online]. Available: https://bit.ly/2QZYP7W

[27] Computers and Structures. (2021) Sap 2000 computer software for structural and earthquake engineering. [Online]. Available: https://bit.ly/2S75M7Q 